\title{
DIVERGENCE OF APPROXIMATING POLYNOMIALS ${ }^{1}$
}

\author{
PHILIP C. CURTIS, JR.
}

I. Introduction. Let $f$ be continuous and periodic on the interval $[0,2 \pi)$. For each integer $n$ let $F_{n}$ be a set of $m_{n}\left(m_{n} \geqq 2 n+1\right)$ points, equally spaced modulo $2 \pi$, from the interval $[0,2 \pi)$. Let $p_{n}(f, x)$ $=a_{0}+\sum_{k=1}^{n}\left(a_{k} \cos k x+b_{k} \sin k x\right)$ be the trigonometric polynomial of order $n$ which approximates $f$ best on $F_{n}$ in the sense of least squares. Let $\|f\|=\sup _{0 \leq x \leq 2 \pi}|f(x)|$. For each continuous $f$ let $P_{n}$ be the operator defined by $\left(P_{n} f\right)(x)=p_{n}(f, x)$. It is easy to verify that $P_{n}$ is linear and idempotent $\left(P_{n}^{2}=P_{n}\right)$. Hence, by a theorem of Nikolaev [2, p. 494]

$$
\left\|P_{n}\right\|=\sup _{\|/\| \leqq 1}\left\|P_{n} f\right\| \geqq \frac{1}{4 \sqrt{ } \pi} \log n .
$$

Applying the uniform boundedness principle one infers that there exists a continuous function $f$ such that $P_{n} f$ fails to converge to $f$ uniformly. This, of course, does not prove that there is a point $x$, and a continuous function $f$ such that $\sup _{n}\left|\left(P_{n} f\right)(x)\right|=\infty$. It is our purpose in this paper to investigate this latter question of pointwise divergence. Call a point $x$ a point of divergence if the above divergence phenomenon occurs at that point. Our two main results can be stated as follows. For each choice of the sets $F_{n}$ the set of points of divergence in $[0,2 \pi)$ is the complement of a set of the first category. If the number of points in $F_{n}$, namely $m_{n}$, satisfies

$$
\underset{n}{\lim \sup } \frac{m_{n}}{n}>\frac{\pi}{\sqrt{ } 2},
$$

then every point $x$ is a point of divergence.

The central tool used to prove both these results is the fact that the approximating operators $P_{n}$ for equally spaced points are translation invariant for a point $x_{n}$, namely $x_{n}=2 \pi / m_{n}$. Indeed, if we denote by $T_{a}$ the operator defined by $\left(T_{a} f\right)(x)=f(x+a) \equiv f_{a}(x), T_{x_{n}} P_{n}$ $=P_{n} T_{x_{n}}$. In this setting the two results may be stated in the following way. Let $Q_{n}$ be a projection from the space $\mathbb{E}$ of continuous periodic functions on $[0,2 \pi)$ onto the space of trigonometric polynomials

Presented to the Society, August 31, 1962 under the title Pointwise divergence of approximating polynomials; received by the editors June 22, 1962.

${ }_{1}^{1}$ This research was supported by the United States Air Force through work done at Space Technology Laboratories, Inc. and under contract AF 49(638)-859. 
of degree $\leqq n$ satisfying $T_{x_{n}} Q_{n}=Q_{n} T_{x_{n}}$ where $x_{n}=2 \pi / m_{n}$ and $m_{n}$ is a positive integer. Then if $\sup _{n} m_{n}=\infty$, points of divergence for the sequence of projections $Q_{n}$ are of the second category. If $\lim \sup _{n} m_{n} / n$ $>\pi / \sqrt{ } 2$, then every point $x$ is a point of divergence.

II. We first state and prove the theorem of Nikolaev for the interval $[0,2 \pi)$. The proof is a slight modification of the one in $[2$, p. 494].

THEOREM 1. Let $Q_{n}$ be a projection of (5) onto the trigonometric polynomials of degree $\leqq n$. Then $\left\|Q_{n}\right\| \geqq(1 / 4 \sqrt{ } \pi) \log n$.

Proof. It is well known [2, p. 88] that for all $n, x$

$$
\left|\sum_{k=1}^{n} \frac{\sin k x}{k}\right| \leqq 2 \sqrt{ } \pi \text {. }
$$

Therefore if

$$
\begin{aligned}
& f(x)=\frac{\cos x}{n}+\cdots+\frac{\cos n x}{1}-\frac{\cos (n+2) x}{1}-\cdots-\frac{\cos (2 n+1) x}{n}, \\
& f(x)=2 \sin (n+1) x \sum_{k=1}^{n} \frac{\sin k x}{k}
\end{aligned}
$$

and $|f(x)| \leqq 4 \sqrt{ } \pi$.

Since $Q_{n}$ is linear and idempotent,

$$
\begin{aligned}
\left(Q_{n} f_{-t}\right)(x)= & \frac{\cos (x-t)}{n}+\cdots+\cos n(x-t) \\
& +\sum_{k=n+2}^{2 n+1}\left(f_{k}(x) \cos k t+g_{k}(x) \sin k t\right)
\end{aligned}
$$

where $f_{k}, g_{k}$ are trigonometric polynomials of degree $\leqq n$. Now

$$
\begin{aligned}
\log n & \leqq \sum_{k=1}^{n} \frac{1}{k}=\frac{1}{2 \pi} \int_{0}^{2 \pi}\left(Q_{n} f_{-t}\right)(t) d t \\
& \leqq \sup _{0 \leqq_{2 \pi}}\left\|Q_{n}\right\|\left\|f_{-t}\right\| \leqq\left\|Q_{n}\right\| 4 \sqrt{ } \pi .
\end{aligned}
$$

Consequently $\left\|Q_{n}\right\| \geqq(1 / 4 \sqrt{ } \pi) \log n$ as required.

Next we verify the translation invariance property for least squares approximating polynomials on sets $F_{n}$ of points equally spaced modulo $2 \pi$. We write $F_{n}=\left\{x_{k}^{(n)}\right\}, k=1, \cdots, m_{n}$, and set $x_{k}^{(n)}=y_{n}$ $+2 \pi k / m_{n}$, where $m_{n} \geqq 2 n+1$. Usually we shall abbreviate $x_{k}^{(n)}$ to $x_{k}$.

LEMma 1. If $P_{n}$ is the least squares approximating operator with re- 
spect to $F_{n}$, then $T_{n_{n}} P_{n}=P_{n} T_{z_{n}}$ where $z_{n}=2 \pi / m_{n}$.

Proof.

$$
\left(P_{n} f\right)(x)=\frac{2}{m_{n}} \sum_{k=1}^{m_{n}} f\left(x_{k}\right) D_{n}\left(x-x_{k}\right) \quad \text { where } \quad D_{n}(x)=\frac{\sin \left(n+\frac{1}{2}\right) x}{2 \sin \frac{1}{2} x} .
$$

Therefore

$$
\begin{aligned}
\left(T_{z_{n}} P_{n} f\right)(x) & =\frac{2}{m_{n}} \sum_{k=1}^{m_{n}} f\left(x_{k}\right) D_{n}\left(x+z_{n}-x_{k}\right) \\
& =\frac{2}{m_{n}} \sum_{k=1}^{m_{n}} f\left(z_{n}+x_{k}\right) D_{n}\left(x-x_{k}\right)=\left(P_{n} T_{z_{n}} f\right)(x) .
\end{aligned}
$$

THEOREM 2. For each $n$ let $Q_{n}$ be a projection from $\sqrt{5}$ onto the trigonometric polynomials of degree $\leqq n$. Suppose for infinitely many positive integers $n_{j}$, there exist integers $m_{j}$ for which $T_{2 \pi / m_{j}} Q_{n_{j}}=Q_{n_{j}} T_{2 \pi / m_{j}}$. If $\sup _{j} m_{j}=\infty$, then the set of points of divergence for the sequence $\left\{Q_{n}\right\}$ is the complement of $a$ set of the first category in $[0,2 \pi)$.

Proof. By further refining the sequence $n_{j}$ we may assume $\lim _{j} m_{j}=\infty$. Suppose the set $S$ of points of convergence is of the second category. If $x \in S$, then $\left(Q_{n} f\right)(x) \rightarrow f(x)$ for each $f \in \mathbb{C}$, and by the uniform boundedness principle

$$
\sup _{n} \sup _{\|f\| \leq 1}\left|\left(Q_{n} f\right)(x)\right|<\infty \text {. }
$$

Setting $S_{k}=\left\{x: \sup _{n} \sup _{\|f\| \leq 1}\left|\left(Q_{n} f\right)(x)\right| \leqq k\right\}$ we have $S_{k}$ is a closed subset of $[0,2 \pi)$, and $S=\bigcup_{k=1}^{\infty} S_{k}$. Therefore $S_{k_{0}}$ contains an open interval $I=(\alpha, \beta)$ for some integer $k_{0}$. Choose $j_{0}$ large enough so that $j>j_{0}$ implies $2 \pi / m_{j}<|\beta-\alpha|$. Then $U_{l=1}^{m_{i}}\left(\left(2 \pi l / m_{j}\right)+I\right)$ covers the interval $[0,2 \pi)$. Now applying the translation invariance hypothesis we have $\sup _{x \in[0,2 \pi)} \sup _{\|\rrbracket\| 1}\left|\left(Q_{n} g\right)(x)\right| \leqq k_{0}$ if $\|f\| \leqq 1$. Hence, $\left\|Q_{n_{j}}\right\| \leqq k_{0}$ for infinitely many integers $n_{j}$ which contradicts the theorem of Nikolaev.

Examples can be given of a sequence of least squares approximating projections $\left\{P_{n}\right\}$ satisfying the conditions of Theorem 2 for which $\left(P_{n} f\right)(x) \rightarrow f(x)$ for all $x$ in a countable dense set. For instance let $\left\{F_{n}\right\}$ be a sequence of subsets of $[0,2 \pi)$ each containing $2 n+1$ points and let $P_{n}$ be the interpolating projection for $F_{n}$. Now if one requires $F_{n} \subset F_{n+1}$ and that the points of $F_{n}$ are equally spaced when $n=1$ $+3+\cdots+3^{k}, k=1,2, \cdots$, then $P_{n} f(x) \rightarrow f(x)$ for each $x \in U F_{n}$; and $\left\{P_{n}\right\}$ satisfies the hypothesis of Theorem 2 .

A natural conjecture in this regard is that the translation invari- 
ance hypothesis can be dropped from Theorem 2 . The author has been unable to prove this, however.

III. In this section we give a sufficient condition for every point of the interval to be a point of divergence for the sequence $\left\{Q_{n}\right\}$. We need first a preliminary result due to Bernstein $[1$, p. 57].

LEMMA 2. Let $F_{n}$ be a finite set of points in $[0,2 \pi)$ such that for each $x \in(0,2 \pi), d\left(x, F_{n}\right) \equiv \inf _{y \in F_{n}}|x-y| \leqq \pi / m_{n} .^{2}$ If $p_{n}$ is a trigonometric polynomial of order $n$, and $m_{n} / n \geqq \lambda>\pi / \sqrt{ } 2$, then

$$
\left\|p_{n}\right\| \leqq \frac{2 \lambda^{2}}{2 \lambda^{2}-\pi^{2}} \sup _{x \in F_{n}}\left|p_{n}(x)\right| .
$$

Proof. Let $M=\left\|p_{n}\right\|$, and $N=\sup _{x \in F_{n}}\left|p_{n}(x)\right|$. Then by a familiar theorem of Bernstein $\left|p_{n}^{\prime \prime}(x)\right| \leqq n^{2} M$. If $x_{0}$ is a point at which $\left\|p_{n}\right\|$ is attained, then $p_{n}^{\prime}\left(x_{0}\right)=0$; and hence

$$
\left|p_{n}^{\prime}(x)\right| \leqq\left|x-x_{0}\right| n^{2} M
$$

and

$$
\left|p_{n}(x)-p_{n}\left(x_{0}\right)\right| \leqq \frac{\left|x-x_{0}\right|^{2}}{2} n^{2} M
$$

Since $d\left(x_{0}, F_{n}\right) \leqq \pi / m_{n}$, this yields

$$
M-N \leqq \frac{\pi^{2} n^{2}}{2 m_{n}^{2}} M,
$$

or

$$
M \leqq \frac{1}{1-\frac{\pi^{2} n^{2}}{2 m_{n}^{2}}} N \leqq \frac{2 \lambda^{2}}{2 \lambda^{2}-\pi^{2}} N
$$

TheOREM 3. For each $n$ let $Q_{n}$ be a projection from (5) onto the trigonometric polynomials of degree $\leqq n$. Suppose for infinitely many positive integers $n_{j}$ there exist integers $m_{j}$ for which

$$
T_{2 \pi / m_{j}} Q_{n_{j}}=Q_{n_{j}} T_{2 \pi / m_{j}} \text {. }
$$

If $\lim \sup _{j} m_{j} / n_{j}>\pi / \sqrt{ } 2$, then all points of $[0,2 \pi)$ are points of divergence.

Proof. Suppose $x_{0}$ is a point of convergence then

2 Here $|x|$ is computed modulo $2 \pi$. 


$$
\sup _{n} \sup _{\|f\| \leqq 1}\left|\left(Q_{n} f\right)\left(x_{0}\right)\right|<\infty .
$$

But if $T_{2 \pi / m_{n}} Q_{n}=Q_{n} T_{2 \pi / m_{n}}$, then

$$
L_{n} \equiv \sup _{\|f\| \leqq 1}\left|\left(Q_{n} f\right)\left(x_{0}\right)\right|=\sup _{\|f\| \leqq 1}\left|\left(Q_{n} f\right)\left(x_{0}+\frac{2 \pi l}{m_{n}}\right)\right|, l=1,2, \cdots, m_{n} .
$$

Applying Lemma 2 to $F_{n}=\left\{x_{0}+\left(2 \pi l / m_{n}\right)\right\}, l=1, \cdots, m_{n}$, when $m_{n} / n \geqq \lambda>\pi / \sqrt{ } 2$, we have that $\left\|Q_{n}\right\| \leqq$ const. $L_{n}$. The hypothesis of the theorem then implies $\sup _{j}\left\|Q_{n_{j}}\right\|<\infty$ for some sequence of integers $\left\{n_{j}\right\}$. This again contradicts the theorem of Nikolaev.

Some rate of growth assumption for $m_{n}$ is necessary in order to guarantee that all points of $[0,2 \pi)$ are points of divergence. For, referring back to the example of $\S I I$, suppose

$$
n_{k} \equiv 1+\cdots+3^{k} \leqq n<1+\cdots+3^{k+1} \equiv n_{k+1} .
$$

Define

$$
Q_{n}=\frac{1}{2 n_{k}+1} \sum_{j=1}^{2 n_{k}+1} T_{2 \pi j /\left(2 n_{k}+1\right)} P_{n} T_{2 \pi j /\left(2 n_{k}+1\right)}^{-1} .
$$

It may be verified easily that $Q_{n}$ is a projection of $\mathbb{S}$ onto the trigonometric polynomials of order $\leqq n . Q_{n}=P_{n}$ if $n=n_{k_{j}}$; and if $n_{k} \leqq n$ $<n_{k+1}, \quad T_{2 \pi /\left(2 n_{k}+1\right)} Q_{n}=Q_{n} T_{2 \pi /\left(2 n_{k}+1\right)}$. Moreover, for $n \geqq n_{k},\left(Q_{n} f\right)(x)$ $=f(x)$ if $x \in F_{n_{k}}$. Therefore $\left(Q_{n} f\right)(x) \rightarrow f(x)$ if $x \in U_{n} F_{n}$.

\section{BIBLIOGRAPHY}

1. S. Bernstein, Sur une modification de la formule d'interpolation de Lagrange Comm. Soc. Math. Kharkov 5 (1931), 49-57.

2. I. P. Natanson, Konstruktive Funktionentheorie, Akademie Verlag, Berlin, 1955.

University of California, Los Angeles 